Check for updates

Cite this: J. Mater. Chem. A, 2019, 7, 6113

Received 25th January 2019

Accepted 19th February 2019

DOI: $10.1039 /$ c9ta00993k

rsc.li/materials-a

\section{Humidity-gated, temperature-responsive photonic infrared reflective broadband coatings $\uparrow$}

\author{
Ellen P. A. van Heeswijk, (D) ab Joey J. H. Kloos, (D) ${ }^{a}$ Nadia Grossiord (D) *c \\ and Albertus P. H. J. Schenning iD *abd
}

The fabrication of temperature responsive photonic polymers remains a challenge. Here, we report the fabrication of humidity-gated temperature-responsive infrared reflective photonic coatings using an easyto-process bar-coating technique. At high humidity the hydroscopic cholesteric liquid crystalline polymer is able to absorb water vapour from the air causing swelling of the photonic coating. By increasing the temperature, water is desorbed from the coating, resulting in a reversible $420 \mathrm{~nm}$ shift of the photonic reflection band. In particular, it is shown that temperature-responsive single-layered broadband IR reflective coatings, prepared by creation of a pitch gradient of the cholesteric liquid crystals, might be suitable for smart window applications in high relative humidity environments such as greenhouses.

\section{Introduction}

Stimuli-responsive photonic materials which are able to change their reflection colour as a function of external stimuli, such as light, temperature and water, are currently receiving a lot of attention. $^{1-6}$ These materials are appealing for a variety of applications such as sensors, ${ }^{7-9}$ security ${ }^{10}$ and energy saving. ${ }^{11,12}$ In the latter case, since IR light from the sun is responsible for heating up buildings, automobiles and greenhouses, photonic materials could be used as temperature responsive infrared (IR) reflective coatings for windows for energy efficient indoor heat control. ${ }^{13}$ For energy efficient windows, a response towards temperature is desired, enabling reflection of heat during warmer periods and transmission of heat (IR) during cooler periods. ${ }^{\mathbf{1 4}}$ To avoid interference with visible light, the reflection band should be able to shift to longer wavelengths upon cooling allowing the highest intensity IR light from the sun (from around $800-1200 \mathrm{~nm}$ ) to transmit though the window and contribute to passive heating. Temperature responsive materials switching between transparent and scattering or absorbing states, such as hydrogels, ${ }^{15,16}$ phase changing materials ${ }^{12,17-19}$ or polymer composite coatings ${ }^{\mathbf{2 0}}$ have been developed for temperature responsive smart window applications. Also static IR reflecting coatings have been studied intensively. ${ }^{13}$ However, the fabrication of temperature responsive photonic polymer coatings focusing on reflection of IR light remains a challenge.
Cholesteric liquid crystals (Ch-LC) are a well-known class of stimuli-responsive photonic materials able to reflect light. ${ }^{21,22}$ The helical molecular organization in these materials acts similarly to Bragg reflectors. Ch-LC are often used for temperature-responsive polymer stabilized systems inside cells ${ }^{23-26}$ or as irreversible time-temperature integrators. ${ }^{27,28} \mathrm{~A}$ coating of a non-crosslinked, oligomeric Ch-LC mixture was recently developed showing a shift of the narrow IR-reflection band to shorter wavelengths upon increasing the temperature. ${ }^{29}$ However, due to non-crosslinked nature of the coating, reaching temperatures lower than $16{ }^{\circ} \mathrm{C}$, resulted in the reflectivity being irreversibly lost. Cross-linked thermochromic photonic systems based on hydrogels ${ }^{30,31}$ have been reported: upon heating or cooling of the surrounding water, reflection bands are able to shift in the visible light range.

We now report on hygroscopic cholesteric liquid crystalline crosslinked IR reflective coatings which display a humiditygated temperature-response. These photonic materials show a shift of the IR reflection band to longer wavelengths upon cooling in atmospheres above $45 \%$ relative humidity $(\mathrm{RH})$, and the working mechanism is elucidated. Moreover, broadband reflecting coatings are prepared showing similar shifts. These coatings can be easily processed by bar coating techniques on flexible polycarbonate (PC) films. Due to the low density, toughness, flexibility, heat resistance, and high impact strength, plastics such as PC are desirable materials for
${ }^{a}$ Stimuli-responsive Functional Materials and Devices, Department of Chemical Engineering, Eindhoven University of Technology, P.O. Box 513, $5600 \mathrm{MB}$ Eindhoven, The Netherlands

${ }^{b}$ Institute for Complex Molecular Systems, Eindhoven University of Technology, P.O. Box 513, 5600 MB Eindhoven, The Netherlands

${ }^{c}$ SABIC, T\&I, Plasticslaan 1, 4612 PX Bergen op Zoom, The Netherlands. E-mail: nadia.grossiord@sabic.com
${ }^{d}$ SCNU-TUE Joint Laboratory of Device Integrated Responsive Materials (DIRM), Guangzhou Higher Education Mega Center, South China Normal University, 510006 Guangzhou, China. E-mail: a.p.h.j.schenning@tue.nl

$\dagger$ Electronic supplementary information (ESI) available: FTIR and UV-Vis spectra, dew point calculations and photographs of samples. See DOI: $10.1039 / \mathrm{c} 9 \mathrm{ta} 00993 \mathrm{k}$ 
applications in windows and roofs in automotive or building and construction markets. ${ }^{32}$ Our temperature responsive broadband reflecting coatings will be highly interesting for autonomous temperature regulation in greenhouses and tropical environments.

\section{Materials and methods}

LEXAN $^{\text {TM }}$ 8040T PC films and a benzophenone-PC copolymer (XPC) films were kindly provided by SABIC IP.\$ Liquid crystalline monomers 1, 3 and 4, depicted in Fig. 1, were purchased from Merck. The chiral dopant 2 was obtained from BASF, benzoic acid derivatives 6 and 7 were prepared by Synthon, the photo-initiator benzophenone (BP), dye $\mathbf{5}$ and potassium hydroxide $(\mathrm{KOH})$ pellets were purchased from Sigma Aldrich and the surfactant 8 was purchased from BOC Sciences. All solvents were obtained from Biosolve and used without further purification.

Two cholesteric mixtures were used in which the overall crosslink density was kept constant. Ch-LC mixture I was used to prepare the coatings with a narrow reflection band (that is, no pitch gradient was formed). Ch-LC mixture II was used to prepare the coatings with the widened reflection band (a pitch gradient was formed). Ch-LC mixture I: $37.7 \mathrm{wt} \% 1,2.5 \mathrm{wt} \% 2$, $14.9 \mathrm{wt} \%$ 3, $21.7 \mathrm{wt} \%$ 6, $21.7 \mathrm{wt} \% 7$ and $1.5 \mathrm{wt} \%$ BP. Ch-LC mixture II: 14.7 wt\% 1, 15.6 wt\% 3, 27.1 wt $\%$ 4, 1.2 wt\% 5, 20.4 wt $\%$ 6, 20.4 wt\% 7, 0.6 wt\% 8.

\section{Fabrication of the narrowband IR reflective Ch-LC coating}

Approximately $30 \mathrm{~cm}^{2}$ LEXAN ${ }^{\mathrm{TM}}$ 8040T PC film was wetted at $40{ }^{\circ} \mathrm{C}$ by $0.5 \mathrm{ml}$ primer solution of $10 \mathrm{wt} \% \mathrm{BP}$ and $90 \mathrm{wt} \%$ ethanol. The ethanol was allowed to evaporate for 15 minutes. Ch-LC mixture I was applied on the pre-treated substrate with a bar-coat applicator having a gap height of $30 \mu \mathrm{m}$ at room temperature (RT) and cured though the substrate at RT for 5 minutes using UV-light with an intensity of $30 \mathrm{~mW} \mathrm{~cm}^{-2}$ in the range between 320 and $390 \mathrm{~nm}$. Afterwards, the fully polymerized Ch-LC coating was submerged in a $1 \mathrm{M} \mathrm{KOH}$ solution for 10 minutes, rinsed with water and then dried by heating the coating to $70{ }^{\circ} \mathrm{C}$ for several minutes.

\section{Fabrication of the broadband IR reflective Ch-LC coating}

Approximately $30 \mathrm{~cm}^{2}$ XPC was wetted by $0.5 \mathrm{ml}$ primer solution of $0.5 \mathrm{wt} \% \mathrm{BP}$ and $99.5 \mathrm{wt} \%$ ethanol at $40{ }^{\circ} \mathrm{C}$. The ethanol was allowed to evaporate for 15 minutes. Ch-LC mixture II was applied on the pre-treated substrate with a bar-coat applicator having a gap height of $30 \mu \mathrm{m}$ at $70{ }^{\circ} \mathrm{C}$ and cured through the substrate at $70{ }^{\circ} \mathrm{C}$ for $15-20$ minutes using UV light with an intensity of $3 \mathrm{~mW} \mathrm{~cm}^{-2}$ in the range of $320-390 \mathrm{~nm}$, followed by a 5 minutes post-cure at $70{ }^{\circ} \mathrm{C}$ using UV-light with an intensity of $30 \mathrm{~mW} \mathrm{~cm} \mathrm{~cm}^{-2}$ in the range of $320-390 \mathrm{~nm}$. Afterwards, the

\$ Copolycarbonate film substrate, comprising a copolycarbonate of bisphenol A and $10 \mathrm{~mol} \%$ of 4,4'-dihydroxybenzophenone, $M_{\mathrm{w}}$ of $21000-23000 \mathrm{~g} \mathrm{~mol}^{-1}$ as determined by GPC using bisphenol A polycarbonate standards, phenol end-capped as described in WO 2015/193862

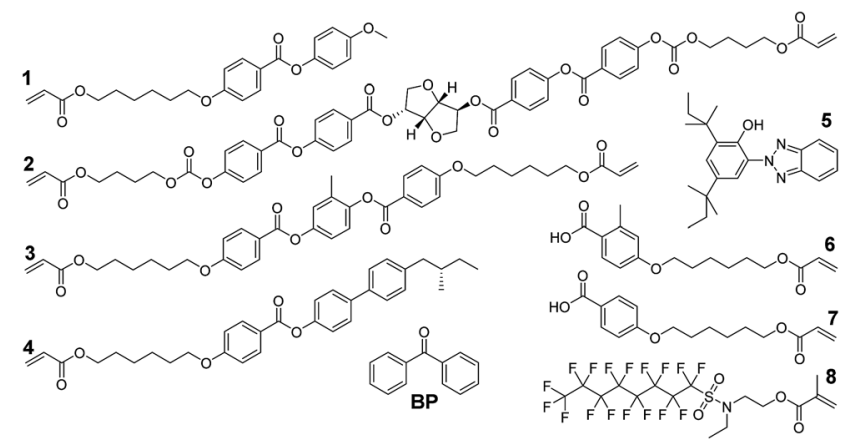

Fig. 1 The chemicals used for the preparation of the Ch-LC polymer networks.

fully polymerized Ch-LC coating was submerged in a $1 \mathrm{M} \mathrm{KOH}$ solution for approximately 30 minutes, rinsed with water and then dried by heating the coating to $70{ }^{\circ} \mathrm{C}$ for several minutes.

\section{Characterization}

Attenuated total reflection Fourier transfer infrared (ATR-FTIR) was measured on a Varian 670-IR equipped with a golden gate setup. UV-Vis spectroscopy was performed on a Perkin Elmer Lambda 750 equipped with a $150 \mathrm{~mm}$ integrating sphere. Haziness of the coating was measured according to the ASTM standard D-1003-00 and was averaged over 10 different locations of the coating. UV-Vis spectroscopy measurements with temperature and humidity control were performed on a Shimadzu UV-3102 PC equipped with a Linkam temperature control stage in a transparent humidity controlled chamber.

All spectra were taken after the coating was held for 5 minutes at the desired temperature for stability of the temperature profile. Scanning electron microscopy (SEM) images were obtained from a Fei Quanta 3D-FEG. The measurement parameters were as follows: acceleration voltage: 5 kilovolt, working distance: $10 \mathrm{~mm}$, and high vacuum. Cross-sections of the sample were prepared by submersion in liquid nitrogen, after which the frozen sample was cut using a razor blade and sputter coated with gold.

\section{Results and discussion}

\section{Fabrication of the narrowband IR reflective Ch-LC coating}

Initially a narrowband hygroscopic Ch-LC coating was prepared to analyse the thermochromic behaviour of the coating. To avoid delamination between the plastic substrate and Ch-LC coating, polycarbonate films were pre-treated with $\mathrm{BP}$ as was described earlier. $^{33}$ The BP type II photo-initiator creates radicals at the substrate interface by hydrogen abstraction, enabling grafting of the acrylate monomers from the substrate. Subsequently, Ch-LC mixture I was coated on the pre-treated polycarbonate using an easy-to-process bar-coating technique (Fig. 2A). Using this approach, the necessary cholesteric alignment of the liquid crystals was generated by shear forces, excluding the need for additional alignment layers. ${ }^{33}$ In order to prepare a hydroscopic polymer, the cured coating was soaked in a potassium hydroxide 


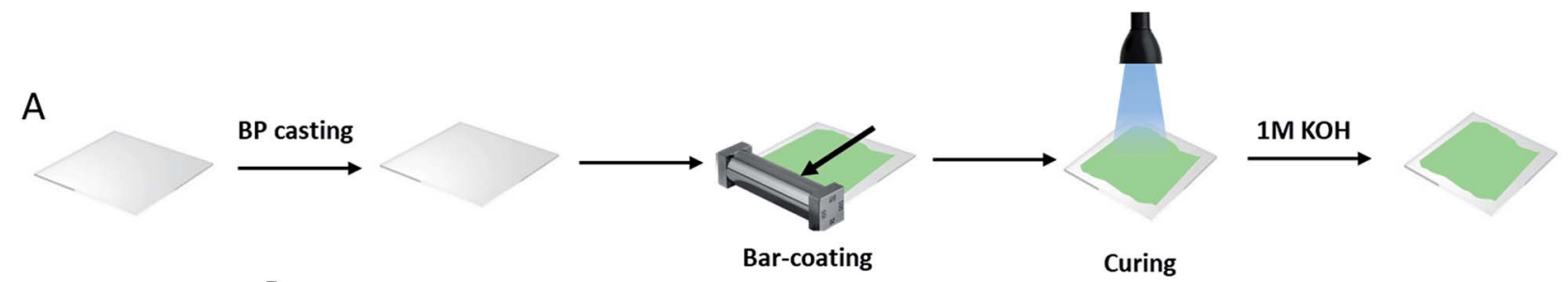

B

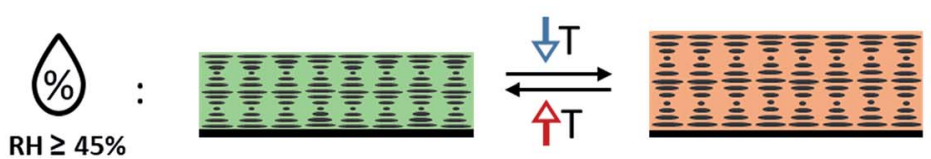

Fig. 2 Preparation method for responsive Ch-LC coatings. (A) Pre-treatment of the substrate with a primer BP-solution, coating using an easyto-process bar-coating technique, followed by bar-coating of the Ch-LC mixture and a photo-polymerization. Finally, the cured coating is exposed to an alkaline treatment to create the hygroscopic Ch-LC coating. (B) Schematic representation of the humidity-gated swelling and deswelling of the Ch-LC coating due to temperature fluctuations.

(KOH) solution to disrupt the hydrogen bonds formed between 6 and/or 7, creating a polymer potassium salt coating able to absorb water (Fig. 2B). FTIR spectra reveals the disruption of the hydrogen bonds (Fig. S1 $†$ ). Due to the pre-treatment with BP, no delamination between the coating and the polycarbonate substrate was observed during submersion of the coating in the alkaline solution. The absorption of water caused the photonic layers to swell, resulting in an approximately $400 \mathrm{~nm}$ red shift of the reflected wavelength. Conversely, drying of the coating induced a blue shift of the Ch-LC reflection band, returning to its original wavelength at $1050 \mathrm{~nm}$.

\section{Humidity-gated temperature-responsive narrowband reflector}

To analyse the thermochromic behaviour of the coating, transmission spectra at various temperatures between 70 to $-2{ }^{\circ} \mathrm{C}$ at $75 \% \mathrm{RH}$ were first taken. By decreasing the temperature of the coating from 30 to $-2{ }^{\circ} \mathrm{C}$ a reversible red shift of $400 \mathrm{~nm}$ from 1150 to $1550 \mathrm{~nm}$ was obtained (Fig. 3A). Due to cooling of the coating, locally the dew point of water vapour in air was reached at $16{ }^{\circ} \mathrm{C}$ according to the extended Magnus equation (eqn (S1), Fig. $\mathrm{S} 2 \dagger$ ). Below this dew point temperature, the absorption rate of water into the hygroscopic coating was increased, owing to condensation of the water vapour from the air. This was evidenced by the drastic increase of the slope of the reflection band shift curve near and below the dew point temperature, approximately between $5{ }^{\circ} \mathrm{C}$ and $15{ }^{\circ} \mathrm{C}$ (Fig. 3B). Upon heating, water was evaporated from the coating, causing the photonic layers to de-swell and the reflection band to return to its original colour.

To ensure complete removal of the water from the coating, the coating was heated to $70{ }^{\circ} \mathrm{C}$ after every cooling/heating cycle. However, as Fig. 3A shows, nearly all water was already removed after reaching a temperature of $30{ }^{\circ} \mathrm{C}$ since the peak present at $1950 \mathrm{~nm}$, which corresponds to the water absorbance, disappears. To analyse whether the temperature-responsive band-shifting behaviour of the coating was solely caused by the absorbance of water, the area of the water absorbance peak was integrated and plotted as a function of the temperature to compare the measured water content with the central position of the reflection band (Fig. 3B). As suggested by the similarity of the trends of the two

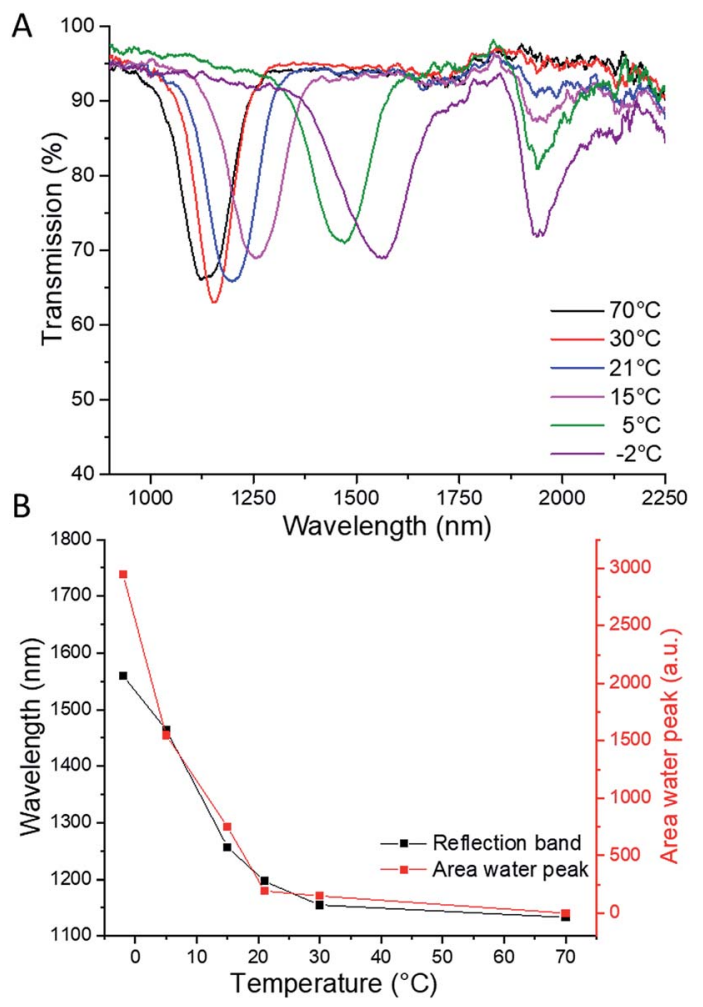

Fig. 3 (A) Transmission spectra of the hygroscopic coating prepared from Ch-LC mixture I, measured at various coating temperatures and a constant environmental $\mathrm{RH}$ of $75 \%$ and temperature of $21^{\circ} \mathrm{C}$. Spectra were measured after the coating had been maintained at the set temperature for 5 minutes. (B) Both the central position of the reflection band and the area of the water absorption peak are plotted vs. temperature.

curves, the origin of the temperature response was primarily governed by water uptake of the hygroscopic coating. Only at low temperatures did the curves deviate significantly from each other. This deviation is most likely caused by the formation of a layer of water at the surface of the coating after saturation of the coating itself. This additional layer of water affects the transmission of the 
IR light (at $1950 \mathrm{~nm}$ ), but does not result in further swelling and thus shift of the reflection band.

To shed light on the influence of the $\mathrm{RH}$ on the thermochromic behaviour of the coating, the temperature response of

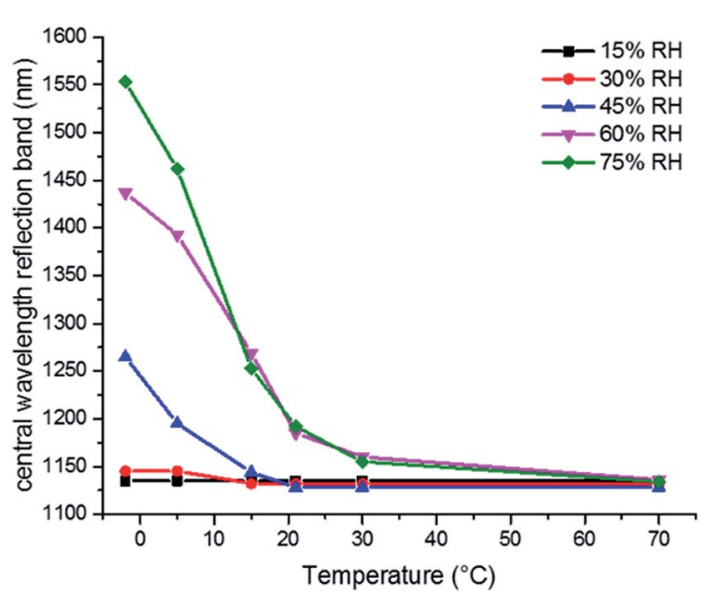

Fig. 4 Central wavelength of the reflection band of the Ch-LC coating measured at different temperatures at various $\mathrm{RHs}$. At $45 \% \mathrm{RH}$, a significant shift of the reflection band is observed. Decreasing the $\mathrm{RH}$ inhibits thermochromic behaviour. Increasing the $\mathrm{RH}$ from $45 \%$ causes an increase in the maximum shift of the reflection band upon temperature variations and an increase in the temperature window in which the coating is responsive. Measurements were taken after maintaining the coating at the given temperature for 5 minutes. the coating was investigated at different RHs (Fig. 4). Below 30\% $\mathrm{RH}$, no significant shift of the reflection band was observed. At $45 \% \mathrm{RH}$, a $137 \mathrm{~nm}$ shift of the reflection band was observed when cooling from $21{ }^{\circ} \mathrm{C}$ to $-2{ }^{\circ} \mathrm{C}$. Increasing the concentration of water vapour in the air resulted in an increase in the maximum shift of the reflection band and an increase of the temperature window within which the coating is responsive. At $60 \% \mathrm{RH}$, a $300 \mathrm{~nm}$ red shift was achieved between $70{ }^{\circ} \mathrm{C}$ to $-2{ }^{\circ} \mathrm{C}$ and at $75 \%$ a maximum shift of $420 \mathrm{~nm}$ between $70{ }^{\circ} \mathrm{C}$ to $-2{ }^{\circ} \mathrm{C}$ was achieved (vide supra). Transmission spectra of the coating at all temperatures and RH studied are included in the ESI (Fig. S3). $\dagger$ This humidity-gated responsive behaviour showing a temperature response above $45 \% \mathrm{RH}$ is most likely due to the decreased dew point at lower RH. For example, at $15 \% \mathrm{RH}$ the dew point is calculated to be $-7{ }^{\circ} \mathrm{C}$ (eqn (S1), Fig. $\mathrm{S} 2 \dagger)$. Therefore, these temperature responsive coatings are especially interesting for heat reflecting windows in higher $\mathrm{RH}$ climates, such as greenhouses. ${ }^{34}$

\section{Fabrication of the broadband IR reflective Ch-LC coating}

To create a broader reflection band, a pitch gradient was created. ${ }^{35}$ By partial diffusion of the photo-initiator from the substrate-coating interface into the coating during photopolymerization, a concentration difference of radicals throughout the thickness of the coating should be created. Due to depletion-induced diffusion of the monomers, this
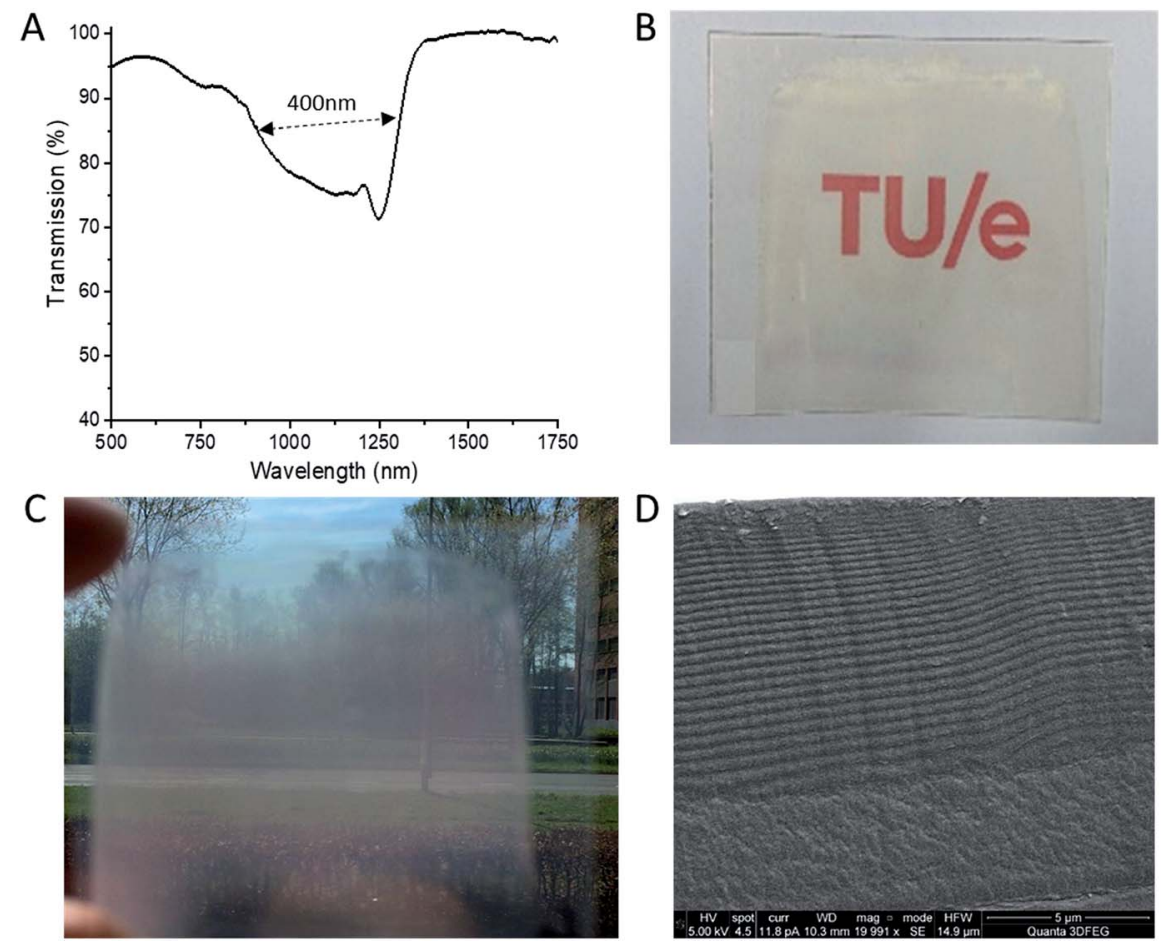

Fig. 5 (A) Transmission spectra of the Ch-LC coating prepared from Ch-LC mixture II, after the alkaline treatment. The hygroscopic coating has a bandwidth of approximately $400 \mathrm{~nm}$, measured at full-width half-maximum as is indicated by the dashed lines. (B) Photograph of the coating and substrate covering the TU/e logo, showing excellent transmission of the visible light. (C) Photograph of the coating and substrate in front of a window, showing the effect of haze. (D) SEM image of the cross-section of the coating. Cholesteric lines are easily visible with the larger pitches at the bottom (substrate interface) and the smaller pitches at the top (air interface). In between the cholesteric aligned film and the substrate, an area of disordered polymer is visualized, which has a constant thickness across the coating. 
inhomogeneity of radicals creates a difference in chiral dopant concentration, needed for broadband formation. ${ }^{36}$ During the diffusion process, faster reacting diacrylic monomers diffuse towards the areas with a higher polymerization rate due to increased local depletion of those monomers by polymerization, while slower reacting mono-acrylic monomers counter diffuse towards areas with a lower polymerization rate. To optimize the broadband formation, some changes were made during the fabrication process to aid monomer diffusion during photo-polymerization (i and ii) and improve adhesion of the coating to the substrate (iii), as described below.

(i) Since the acidic LCs 6 and 7 were still hydrogen-bonded during the polymerization reaction, their reactivity was comparable to that of a diacrylic monomer. In order to sufficiently differentiate between the reactivity of the various monomers, the diacrylic chiral dopant 2 was exchanged for a mono-acrylic chiral dopant $\mathbf{4}$, keeping the crosslink density constant by adjusting the mono-acrylic versus diacrylic ratio.

(ii) The ability to obtain a gradient in polymerization rate and a widened reflection band by partial diffusion of the photoinitiator BP from the substrate interface into the coating had previously been demonstrated. ${ }^{33}$ To establish enough time for diffusion of the monomers, the photo-curing intensity was lowered by a factor of 10 , the initial concentration of BP applied during pre-treatment was lowered by a factor of 20 and no BP was present in the Ch-LC mixture. Moreover, dye $\mathbf{5}$ was added to enhance the light intensity gradient, amplifying the polymerization rate gradient. ${ }^{36}$ At the substrate interface, the concentration of radicals in the coating was high with respect to the airinterface. Therefore, the diacrylate 3 and the acidic LCs 6 and 7 could diffuse towards the substrate, leaving room for the monoacrylic chiral dopant to counter diffuse towards the coating-air interface. Using this approach, a widened reflection band was created (Fig. S4†).

(iii) Lowering of the BP concentration was needed to ensure a low enough reaction rate so that the monomers were able to sufficiently diffuse prior to polymerization. However, adhesion between the coating and the substrate was compromised when the BP concentration in the primer solution was lowered to $0.5 \mathrm{wt} \%$. To ensure sufficient adhesion of the coating to the substrate after base treatment, PC-benzophenone copolymer XPC was used as a substrate, which contained $10 \mathrm{~mol} \%$ covalently bound non-diffusible BP groups.

After polymerization, similar to the procedure used to prepare the narrowband reflectors, the coating was soaked in an alkaline solution to disrupt the hydrogen bonds. Fig. 5A shows the transmission spectra of the $400 \mathrm{~nm}$-broad reflection band of the Ch-LC coating after $\mathrm{KOH}$ treatment. In comparison with the bandwidth of the Ch-LC coating without a pitch gradient (Fig. 3A), the bandwidth had increased by a factor of 3.3. Fig. 5B and $\mathrm{C}$ show photographs of the coating covering the TU/e logo and the view through a window to the outside, respectively. The TU/e logo is clearly visible behind the coating showing excellent transmission of the broadband coating for visible light. Looking outside the window though the coating, haze $(40 \% \pm 3 \%$, measured by UV-Vis) caused by forward scattering was observed. Scanning electron microscopy (SEM) of a coating cross-section (Fig. 5D) clearly shows a pitch gradient, with the largest pitches near the substrate interface as expected, due to the lower concentration of chiral dopant. In addition to the wellordered cholesteric lines displaying few defects, a non-aligned layer sandwiched between the aligned LCN and the substrate was observed. This non-aligned layer most likely causes the haziness of the coating. BP diffusion through the coating is likely to cause Ch-LC alignment disruption during photopolymerization, because the concentration of this nonmesogenic molecule is too high. The haziness limits these coatings for smart window applications in office buildings, for example, since the outside view would be adversely affected. However, for applications in the greenhouse industry, windows that generate more diffuse light (via forward scattering) are desired for optimal crop growth. In this case, diffuse light penetrates through the leaf canopy to the middle layers of the crop, resulting in increased photosynthesis. ${ }^{37}$

At a fixed $75 \% \mathrm{RH}$, transmission spectra were taken at various temperatures to analyse the coating's thermochromic behaviour (Fig. 6A). The central position of the widened reflection band had an approximate red shift of $400 \mathrm{~nm}$ upon decrease of the temperature from $30{ }^{\circ} \mathrm{C}$ to $2{ }^{\circ} \mathrm{C}$ (Fig. 6B). Note

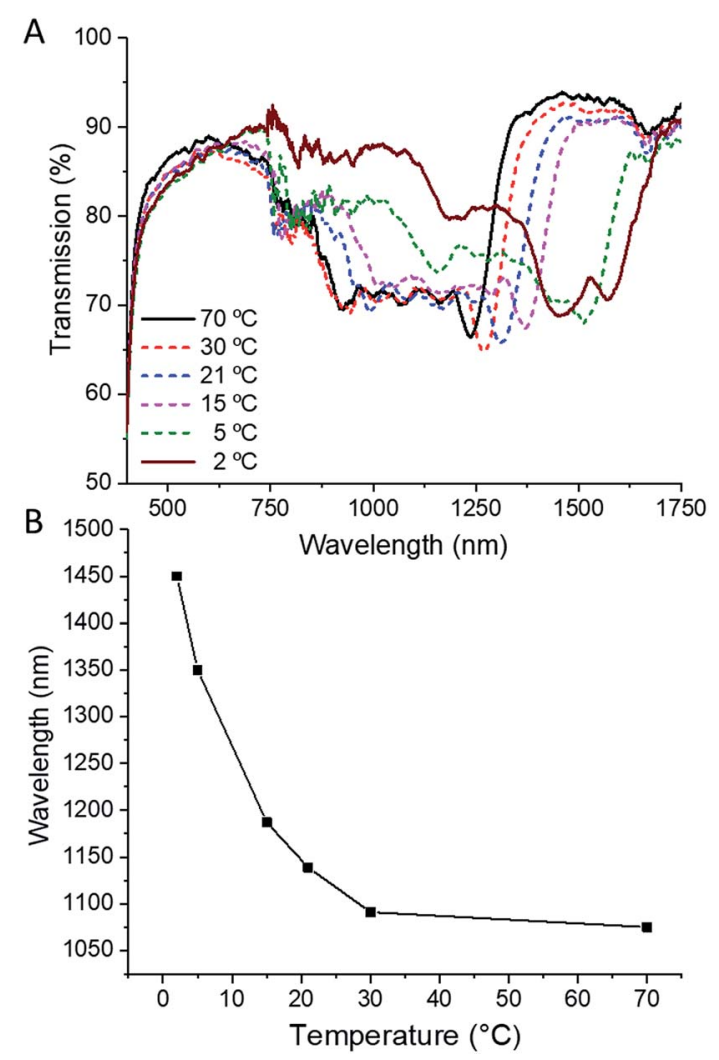

Fig. 6 (A) Transmission spectra of the broadband Ch-LC coating at $75 \% \mathrm{RH}$ and various temperatures. Spectra were measured after the temperature of the coating had been maintained for 5 minutes. (B) The central wavelength of the reflection band plotted as a function of temperature. Note that due to the irregular shape of the reflection band at lower temperatures, the central position is difficult to determine by the full-width half-maximum method, since the width of the reflection band is ill defined. 
that this temperature-triggered red shift is similar to the shift exhibited by the narrowband reflector shown in Fig. 3A. Alongside the red shift of the reflection band upon cooling, the shape of the reflection band was also affected by the temperature: upon cooling from 15 to 5 and $2{ }^{\circ} \mathrm{C}$, the short wavelength portion of the reflection band displays an intensity reduction, due to a loss of the Ch-LC alignment.

The loss of alignment for the smaller pitches is caused by the gradient of the local cross-link density coming from the enrichment in diacrylic (resp. mono-acrylic) monomers towards the coating-substrate interface (resp. towards the coating-air interface). Due to the mono-acrylic nature of the chiral dopant, the smallest pitches are formed in the area of lower crosslink density. Therefore, these smaller pitches showed a greater decline in molecular order upon swelling with respect to the more cross-linked areas of the higher pitches.

To analyse the time needed for the coating to absorb water from the environment, the coating was rapidly cooled down from $70{ }^{\circ} \mathrm{C}$ to $5{ }^{\circ} \mathrm{C}$ and kept isothermal. Afterwards, the coating was rapidly heated to $70{ }^{\circ} \mathrm{C}$ to assess the time needed for desorption of the water present in the coating. The $\mathrm{RH}$ was kept constant at $75 \%$ throughout the entire cycle. Fig. 7A shows the central wavelength shifts of the broadened reflection band during the isothermal stages. In the first 10 minutes, the coating absorbs most of the water, as shown by the rapid reflection band shift from approximately 1080 to $1325 \mathrm{~nm}$. After

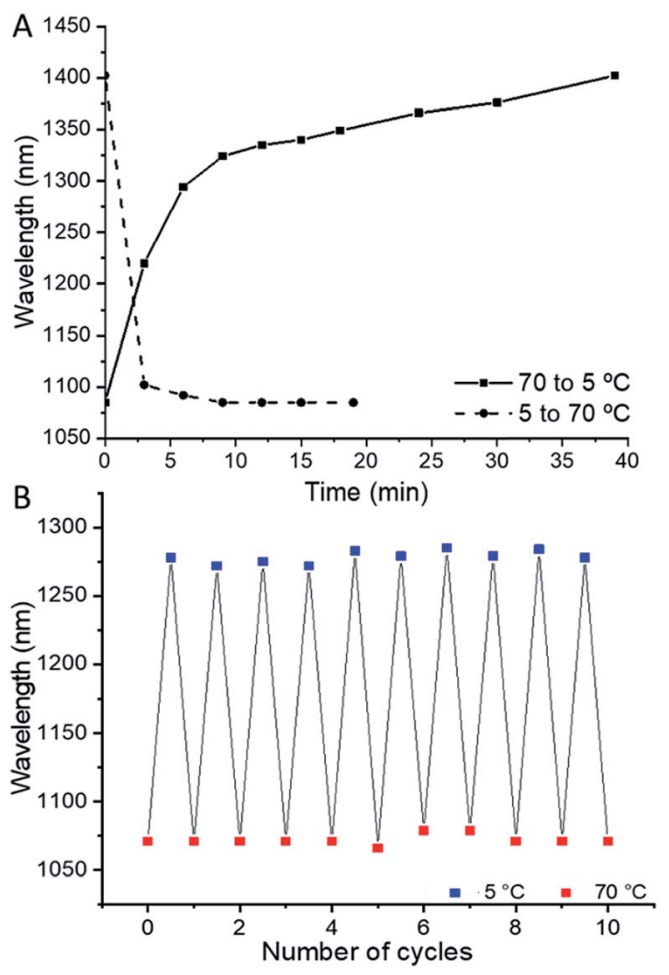

Fig. 7 (A) Central wavelength of the broad reflection band after the coating was heated (dash) or cooled (solid) $50{ }^{\circ} \mathrm{C}$ min $^{-1}$ and kept isothermal at $75 \% \mathrm{RH}$. Spectra were measured every 3 minutes with $t=$ 0 at the start of the cooling or heating ramp. (B) Central wavelength of the reflection band after keeping the coating isothermal for 5 minutes during 10 cycles of heating and cooling at $75 \% \mathrm{RH}$. these initial 10 minutes, the reflection band slowly continues to absorb water, causing disorder in the smaller Ch-LC helix pitch, shifting therefore the central position of the reflection band further (Fig. S5 $\dagger$ and 6A). After heating from $5{ }^{\circ} \mathrm{C}$ to $70{ }^{\circ} \mathrm{C}$, the central wavelength recovered its original position within a few minutes. Alternating heating and cooling of the coating for at least 10 times at $75 \% \mathrm{RH}$ shows good reversibility of the shift of the IR reflection band (Fig. 7B).

\section{Conclusions}

The successful fabrication process for humidity-gated temperature-responsive coatings using an easy-to-process barcoating technique was reported and the working mechanism of its responsive behaviour was elucidated. At $75 \% \mathrm{RH}$, heating or cooling of the coatings result in a blue or red shift of the ChLC reflection band of a maximum of $420 \mathrm{~nm}$, respectively. This temperature-responsive shift is caused by the absorbance of water vapour from the air into the coating at lower temperatures, swelling the photonic layers.

Furthermore, a Ch-LC broadband was created by diffusion of the photo-initiator from the substrate into the polymerizing coating. For these coatings, a $\sim 400 \mathrm{~nm}$ band shift was obtained as well, demonstrating the ability to reflect heat at higher temperatures, while allowing heat to be transmitted through the coating at lower temperatures. These coatings are potential candidates for heat-regulating windows in climates where the humidity is higher than $60 \%$, such as tropical environments and greenhouses. Furthermore, modifying the chiral dopant concentration to a (narrow) reflection band in the visible light, aesthetic or other humidity-gated temperature-responsive applications can be considered as well.

\section{Conflicts of interest}

There are no conflicts to declare.

\section{Acknowledgements}

We acknowledge funding for this project by SABIC. Furthermore, the authors would like to thank Simon Houben for his support with the SEM measurements and Fabio di Lena, Ramon Groote, Theo Hoeks and Michael Debije for their valuable insights.

\section{Notes and references}

1 Q. Li, Intelligent Stimuli Responsive Materials: From WellDefined Nanostructures to Applications, Hoboken, New Jersey, 2013.

2 T. J. White, M. E. McConney and T. J. Bunning, J. Mater. Chem., 2010, 20, 9832-9847.

3 C. I. Aguirre, E. Reguera and A. Stein, Adv. Funct. Mater., 2010, 20, 2565-2578.

4 J. E. Stumpel, D. J. Broer and A. P. H. J. Schenning, Chem. Commun., 2014, 50, 15839-15848.

5 J. Ge and Y. Yin, Angew. Chem., 2011, 50, 1492-1522. 
6 L. Wang and Q. Li, Adv. Funct. Mater., 2016, 26, 10-28.

7 Y. Fang, Y. Ni, S. Y. Leo, C. Taylor, V. Basile and P. Jiang, Nat. Commun., 2015, 6, 1-8.

8 Y. Fang, Y. Ni, S. Y. Leo, B. Wang, V. Basile, C. Taylor and P. Jiang, ACS Appl. Mater. Interfaces, 2015, 7, 23650-23659.

9 D. J. Mulder, A. P. H. J. Schenning and C. W. M. Bastiaansen, J. Mater. Chem. C, 2014, 2, 6695-6705.

10 M. Moirangthem, T. A. P. Engels, J. Murphy, C. W. M. Bastiaansen and A. P. H. J. Schenning, ACS Appl. Mater. Interfaces, 2017, 9, 32161-32167.

11 J. Lu, W. Gu, J. Wei, W. Zhang, Z. Zhang, Y. Yu, N. Zhou and X. Zhu, J. Mater. Chem. C, 2016, 4, 9576-9580.

12 X. Liang, S. Guo, S. Guo, M. Chen, C. Li, Q. Wang, C. Zou, C. Zhang, L. Zhang and H. Yang, Mater. Horiz., 2017, 4, 878-884.

13 H. Khandelwal, A. P. H. J. Schenning and M. G. Debije, Adv. Energy Mater., 2017, 7, 1-18.

14 H. Khandelwal, R. C. G. M. Loonen, J. L. M. Hensen, M. G. Debije and A. P. H. J. Schenning, Sci. Rep., 2015, 5, 11773.

15 Y. Zhou, Y. Cai, X. Hu and Y. Long, J. Mater. Chem. A, 2014, 2, 13550-13555.

16 Y. S. Yang, Y. Zhou, F. B. Yin Chiang and Y. Long, RSC Adv., 2016, 6, 61449-61453.

17 S. Grynning, F. Goia and B. Time, Energy Procedia, 2015, 78, 85-90.

18 A. Seeboth, R. Ruhmann and O. Mühling, Materials, 2010, 3, 5143-5168.

19 L. Wang, H. K. Bisoyi, Z. Zheng, K. G. Gutierrez-Cuevas, G. Singh, S. Kumar, T. J. Bunning and Q. Li, Mater. Today, 2017, 20, 230-237.

20 F. Guo, S. Chen, Z. Chen, H. Luo, Y. Gao, T. Przybilla, E. Spiecker, A. Osvet, K. Forberich and C. J. Brabec, Adv. Opt. Mater., 2015, 3, 1524-1529.

21 N. Tamaoki, Adv. Mater., 2001, 13, 1135-1147.
22 S. T. Kim and H. Finkelmann, Macromol. Rapid Commun., 2001, 22, 429-433.

23 M. E. McConney, V. P. Tondiglia, J. M. Hurtubise, L. V. Natarajan, T. J. White and T. J. Bunning, Adv. Mater., 2011, 23, 1453-1457.

24 Y. Li, Y. Liu and D. Luo, Opt. Express, 2017, 25, 26349.

25 H. Yang, K. Mishima, K. Matsuyama, K. I. Hayashi, H. Kikuchi and T. Kajiyama, Appl. Phys. Lett., 2003, 82, 2407-2409.

26 L. V. Natarajan, J. M. Wofford, V. P. Tondiglia, R. L. Sutherland, H. Koerner, R. A. Vaia and T. J. Bunning, J. Appl. Phys., 2008, 103, 093107.

27 D. J. D. Davies, A. R. Vaccaro, S. M. Morris, N. Herzer, A. P. H. J. Schenning and C. W. M. Bastiaansen, Adv. Funct. Mater., 2013, 23, 2723-2727.

28 M. Moirangthem, J. E. Stumpel, B. Alp, P. Teunissen, C. W. M. Bastiaansen and A. P. H. J. Schenning, Proc. SPIE, 2016, 9769, 97690Y.

29 W. Zhang, S. Kragt, A. P. H. J. Schenning, L. T. De Haan and G. Zhou, ACS Omega, 2017, 2, 3475-3482.

30 Y. Takeoka and M. Watanabe, Langmuir, 2003, 19, 91049106.

31 M. Chen, L. Zhou, Y. Guan and Y. Zhang, Angew. Chem., Int. Ed., 2013, 52, 9961-9965.

32 D. Katsamberis, K. Browall, C. Iacovangelo, M. Neumann and H. Morgner, Prog. Org. Coat., 1998, 34, 130-134.

33 E. P. A. Van Heeswijk, J. J. H. Kloos, J. De Heer, T. Hoeks, N. Grossiord and A. P. H. J. Schenning, ACS Appl. Mater. Interfaces, 2018, 10, 30008-30013.

34 C. Stanghellini, J. Dai and F. Kempkes, Biosyst. Eng., 2011, 110, 261-271.

35 D. J. Broer, J. Lub and G. N. Mol, Nature, 1995, 378, 467-469.

36 D. J. Broer, G. N. Mol, J. A. M. M. Van Haaren and J. Lub, Adv. Mater., 1999, 11, 573-578.

37 S. Hemming, T. Dueck, J. Janse and F. van Noort, Acta Hortic., 2008, 801, 1293-1300. 